\title{
Congrès International d'Histoire de la Pharmacie
}

Paris, 24-29 septembre 1973

Le prochain Congrès International d'Histoire de la Pharmacie, organisé par la Société d'Histoire de la Pharmacie (S.H.P.) sous les auspices de l'Union Mondiale des Sociétés d'Histoire Pharmaceutique (U.M.S.H.P.), de l'Internationale Gesellschaft für Geschichte der Pharmazie (I.G.G.P.) et de l'Académie Internationale d'Histoire de la Pharmacie, se tiendra à Paris en 1973.

Date prévue: 24 au 29 septembre 1973.

Lieu: Paris, Faculté de Pharmacie.

Thème du Congrès : voir ci-dessous.

Langues officielles: français, allemand, anglais.

Des réceptions, visites et excursions sont prévues, notamment: à l'Hôpital Militaire et au Musée du Val-de-Grace; à Chantilly (château, apothicairerie, démonstration équestre) et à Luzarches (église Saint-Côme et Saint-Damien). Un programme spécial sera organisé pour les personnes accompagnantes.

Frais de participation, banquet d'adieu non compris : membres participants $300 \mathrm{~F}$; personnes accompagnantes $200 \mathrm{~F}$ (sauf fluctuations monétaires importantes).

Renseignements, inscriptions et secrétariat :

Congrès International d'Histoire de la Pharmacie

M. Lanchy, 75, rue Ordener, 75018-Paris - Tél. 606-42-57

I. Thème spécial du Congrès

Les relations pharmaceutiques entre la France et les autres pays à travers les siècles.

\section{Communications libres}

A. Histoire générale de la pharmacie, y compris les études biographiques :
1. Des origines au XV $\mathrm{XV}^{\mathrm{e}}$ siècle.
2. $\mathrm{Du} \mathrm{XVI}^{\mathrm{e}}$ au XVIII ${ }^{\mathrm{e}}$ siècle.
3. Du XIX ${ }^{\mathrm{e}}$ siècle à nos jours. 
B. Histoire de la profession pharmaceutique :

1. Organisation et réglementation de la profession.

2. Formation et enseignement pharmaceutiques.

3. Exercice de la profession.

C. Histoire du médicament et des produits pharmaceutiques :

1. Matière médicale.

2. Recherche - Préparation - Fabrication.

3 . Commerce et distribution.

4. Réceptaires - Pharmacopées - Formulaires.

D. Histoire de la pharmacie:

1. Hospitalière.

2. Navale.

3. Militaire.

E. La pharmacie et l'art; les musées.

F. Autres sujets.

Supplément à la «Revue d'Histoire de la Pharmacie» $n^{\circ} 213$ et suivants. 
Gesnerus erscheint halbjährlich in zwei Doppelheften. Preis des Doppelheftes Fr. 26.-, des Jahrganges im Abonnement Fr. 46.-. Mitglieder der Schweizerischen Gesellschaft für Geschichte der Medizin und der Naturwissenschaften erhalten die Zeitschrift gratis (Mitgliederbeitrag Fr. 30.-, Postscheck 80-25315).

Gesnerus paraît deux fois par an. Prix du fascicule double Fr. 26.-, abonnement annuel Fr. 46.-- Les membres de la Société suisse d'histoire de la médecine et des sciences naturelles reçoivent la revue gratuitement (cotisation Fr. 30.-, compte de chèques postaux $80-25315)$.

\section{Mitgliedschaft}

Anmeldung beim Sekretariat (Prof. Dr. med. H.M.Koelbing) Medizinhistorisches Institut der Universität Zürich, Rämistraße 71, 8006 Zürich, oder bei einem andern Vorstandsmitglied. Die Mitglieder erhalten die Veröffentlichungen der Gesellschaft zu einem Vorzugspreis (siehe in diesem Heft Verzeichnis dieser Publikationen). Die Mitglieder sind zur Teilnahme an allen Veranstaltungen der Gesellschaft berechtigt, besonders an der Jahresversammlung, die im Rahmen der Tagung der Schweizerischen Naturforschenden Gesellschaft (s NG) abgehalten wird.

\section{Membres}

Inscription au secrétariat (Prof. Dr. med. H.M. Koelbing) Medizinhistorisches Institut der Universität Zürich, Rämistrasse 71, 8006 Zürich, ou par un membre du comité. Les membres reçoivent les Publications de la Société à un prix réduit (voir la liste des Publications encore disponibles dans ce fascicule). Les membres sont invités à assister à toutes les manifestations de la Société, en particulier à l'assemblée annuelle qui a lieu dans le cadre de la réunion annuelle de la Société helvétique des sciences naturelles (SHSN).

\section{Manuskripte}

Druckfertige Manuskripte in deutscher, französischer, italienischer oder englischer Sprache sind an den Redaktor, Prof. Dr. H.Fischer, Witellikerstraße 60, 8702 Zollikon (Zürich), zu richten.

\section{Manuscrits}

Les manuscrits en français, allemand, italien ou anglais sont à adresser au rédacteur, Prof. Dr. H.Fischer, Witellikerstrasse 60, 8702 Zollikon.

\section{Vorstand / Comité}

Präsident: Prof. Dr. med. Heinrich Bueß, Basel

Vizepräsident: Prof. Dr. med. Erwin H.Ackerknecht, Zürich

Sekretär/Kassier: Prof. Dr. med. Huldrych M. Koelbing, Zürich

Redaktor des Gesnerus: Prof. Dr. med. Hans Fischer, Zollikon

Beisitzer: Dr. phil. Heinz Balmer, Konolfingen (Bern)

Dr. med. Pietro Eichenberger, Münsingen (Bern)

Publiziert mit Unterstützung der Schweizerischen Naturforschenden Gesellschaft

Publié avec l'aide de la Société suisse des sciences naturelles 